\title{
Analysis of the time-of-flight neutron scattering cross-section data for light water measured at the SEQUOIA spectrometer, Spallation Neutron Source (SNS)
}

\author{
Vaibhav Jaiswal ${ }^{1, *}$, Luiz Leal $^{1}$, and Alexander I. Kolesnikov ${ }^{2}$ \\ ${ }^{1}$ Institut de Radioprotection et de Sûreté Nucléaire PSN-EXP/SNC/LNR, Fontenay-aux-Roses, 92260, France \\ ${ }^{2}$ Neutron Scattering Sciences Division, Oak Ridge National Laboratory, Oak Ridge, TN 37831-6473, USA
}

\begin{abstract}
Thermal neutron scattering cross-section data for light water available in the major nuclear data libraries observes significant differences especially at reactor operating temperatures. During the past few years there has been a renewed interest in reviewing the existing thermal scattering models and generating more accurate and reliable thermal scattering cross sections using existing experimental data and in some cases based on Molecular Dynamics (MD) simulations. There is a need for performing new time-of-flight experiments at high temperatures and pressures, to have a better understanding of the physics involved in the scattering process that could help improve the existing TSL data.

Lack of experimental thermal scattering data for light water at high temperatures led to a new measurement campaign within the INSIDER project at the Institut de radioprotection et de sûreté nucléaire (IRSN). Double differential scattering cross section for light water have been measured at the SEQUOIA spectrometer based at the Spallation Neutron Source (SNS), Oak Ridge National Laboratory, United States. Several measurements have been carried out at different temperatures and pressures corresponding to liquid light water. Measurements at five different incident neutron energies $E_{i}(8,60,160,280$ and $800 \mathrm{meV})$ have been carried out to help exploring different regions of the frequency spectrum. This paper presents the analysis of the dynamic structure factor and the derived frequency spectrum of light water. The analysis of the experimental data would provide one with better confidence, the behavior of thermal scattering cross sections for light water at high temperatures, knowledge of which is very important for the design of novel reactors as well as existing pressurized water reactors.
\end{abstract}

\section{Introduction}

Reliable thermal scattering cross-section data, often termed as Thermal Scattering Law (TSL) or $S(\alpha, \beta)$ for light water are important for reactor physics and criticality safety applications. Pressurized Water Reactors (PWRs) operate with light water at high temperature and pressure, i.e., around $550 \mathrm{~K}$ and 150 bar. Hence, an accurate estimation of TSL both at the room temperature and reactor operating temperature is essential. The two most recent TSL library for light water that are widely used are the one in the JEFF-3.3 [1], based on time-of-flight (TOF) experiments measured in the 60s [2] and a recent one available in the ENDF/B-VIII.0, based on Molecular Dynamics (MD) simulations using GROMACS and TIP4P/2005f water potential [3]. Irrespective of the TOF approach or MD simulation approach, TSLs are generated using the LEAPR module of the NJOY code [4] that takes frequency spectrum of the material as an input. It is thus vital to correctly quantify the behavior of the frequency spectrum of light water at the required temperatures and pressures.

Certain differences have been observed in the frequency spectrum obtained using TOF experiments and the

*e-mail: vaibhav.jaiswal@irsn.fr
MD simulations at reactor operating temperatures in both JEFF-3.3 and ENDF/B-VIII.0 TSL library [5]. A major difference is the variation of the energy of the rotation band with increasing temperature in the ENDF/B-VIII.0. This shift of the peak of the rotation band towards lower energies is not present in the JEFF-3.3 frequency spectrum as can be seen in Fig. 1. Also, both these libraries replace the bending and the stretching mode (asymmetric and symmetric) of the frequency spectrum with discrete oscillators while modeling the TSL using LEAPR. Both these libraries use discrete oscillator for bending mode at 205 $\mathrm{meV}$ for all the temperatures and for the stretching mode JEFF-3.3 uses an oscillator at $436 \mathrm{meV}$ and ENDF/BVIII.0 at $410 \mathrm{meV}$ for all the temperatures. Variation of the energy of these oscillators is still unknown due to lack of experimental data and it is assumed that it does not vary as a function of temperature.

Presently, neither the available TOF experiments nor the MD simulations are fully sufficient to generate reliable TSL data for light water at reactor operating conditions. For the case of TOF experiments, there is a lack of sufficiently high-resolution experimental data in the required temperature and pressure range. The accuracy of MD simulations depends on the classical interacting po- 

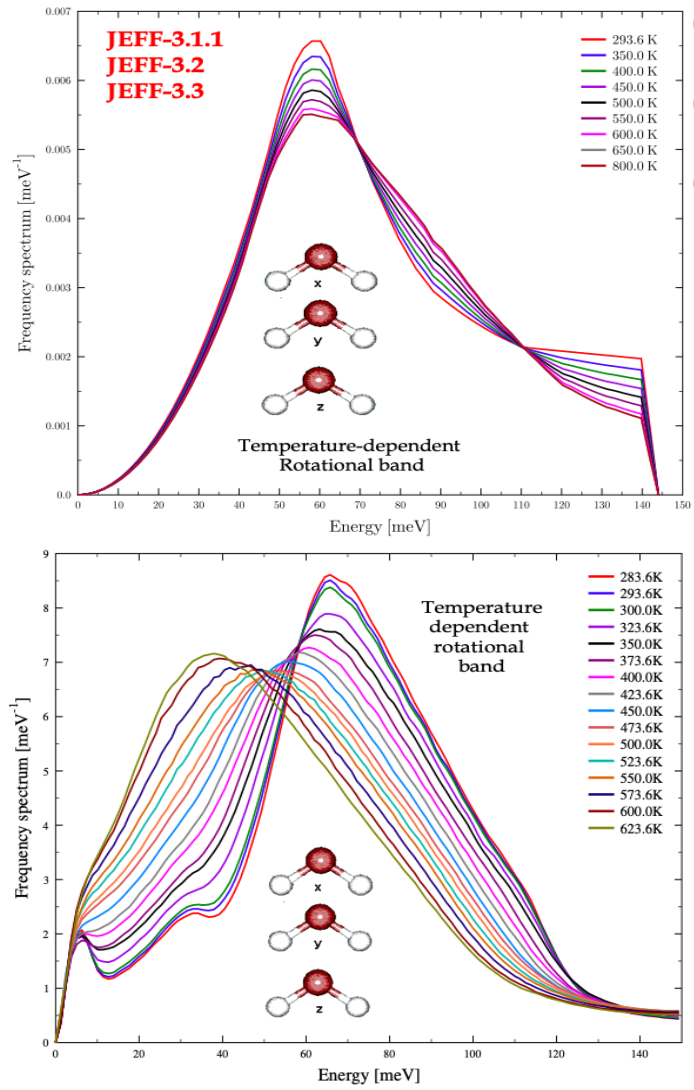

Figure 1. Rotation band of the frequency spectrum of light water as a function of temperature as in JEFF-3.3 (top) and ENDF/B-VIII.0 (bottom) TSL library [1,3].

tentials valid throughout reactor operating temperatures. It has been seen that not all the properties of light water can be reproduced at high temperatures using water potentials that were parameterized for room temperatures [6]. Also, in the present nuclear data libraries, TSLs are considered only temperature-dependent and pressure is not taken into account when generating TSLs, and hence, no information exists on the impact of pressure on the TSL for light water.

The goal of this paper is not to present a new TSL for light water, instead present reliable high-resolution experimental frequency spectrum to better understand the physics behind the different energy modes in the spectrum, not only at room temperature but also at reactor operating temperatures. This would help answering the discrepancies observed in the TSL available in the JEFF-3.3 and ENDF/B-VIII.0 and help developing accurate TSL for light water in the future.

\section{Methodology}

A series of experiments were carried out at different incident energies of the neutron $E_{i}$, in order to inspect various features of the frequency spectrum of light water. Three measurements were made for each temperature, one with the sample and the cell holder, one with the empty cell holder, and the third one with a Vanadium sample for the data normalization. Table 1 shows the thermodynamic conditions of the TOF measurements at different temperatures and pressures. Details about the experimental setup is presented in [7]. The experimental data was corrected

Table 1. Thermodynamic conditions of the measurements for light water

\begin{tabular}{llll}
\hline Sample & $\begin{array}{l}\text { Temperature } \\
(\mathrm{K})\end{array}$ & $\begin{array}{l}\text { Pressure } \\
(\text { bar })\end{array}$ & $\begin{array}{l}\text { Incident neutron } \\
\text { energy }(\mathrm{meV})\end{array}$ \\
\hline \multirow{4}{*}{ Water } & 295 & 1 & $60,160,280,800$ \\
+ & 323 & 1 & $60,160,280,800$ \\
Cell & 350 & 1 & $12,60,160,280,800$ \\
& 450 & 150 & $8,60,160,280,800$ \\
& 450 & 150 & $8,60,160,280,800$ \\
& 500 & 150 & $8,60,160,280,800$ \\
& 550 & 150 & $8,60,160,280,800$ \\
Cell & 600 & 150 & $8,60,160,280,800$ \\
& 295,350, & 150 & $8,60,160,280,800$ \\
\hline Vanadium & 295 & 1 & $8,60,160,280,800$ \\
\hline
\end{tabular}

using the the DAVE scientific package [8] to obtain the dynamic structure factor $S(q, \omega)$. Figure 2 shows an example of the corrected $S(q, w)$ for light water obtained at temperature, $550 \mathrm{~K}$, and pressure, 150 bar. $S(q, \omega)$ obtained

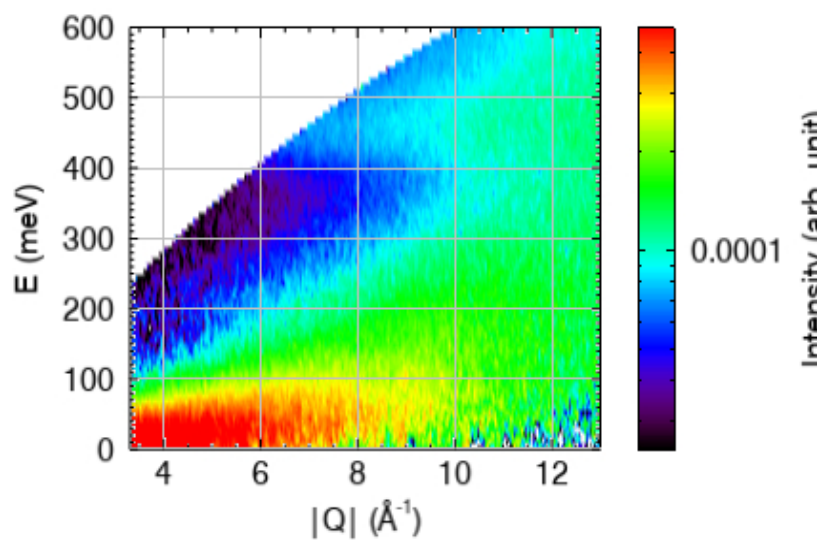

Figure 2. Dynamic structure factor, $S(q, \omega)$ for light water at $500 \mathrm{~K}$ and 150 bar, with $E_{i}$ as $800 \mathrm{meV}$. $Q$ denotes the momentum transfer and $E$ denotes the energy transfer

at different temperatures and pressures is then transformed to the frequency spectrum.

\section{Results}

\subsection{Pressure dependence on the frequency spectrum}

The pressure dependence on the frequency spectrum of light water has been demonstrated by analyzing the same temperature $(350 \mathrm{~K})$ data at different pressures $(1$ bar and 150 bar). The reason behind the choice of this pressure range was to study if the reactor pressure has an impact 
on the TSL or the frequency spectrum of light water. Figure 3 shows the frequency spectrum at different incident energies and the corresponding vibrational modes accessible due to the choice of this energy. The uppermost plot
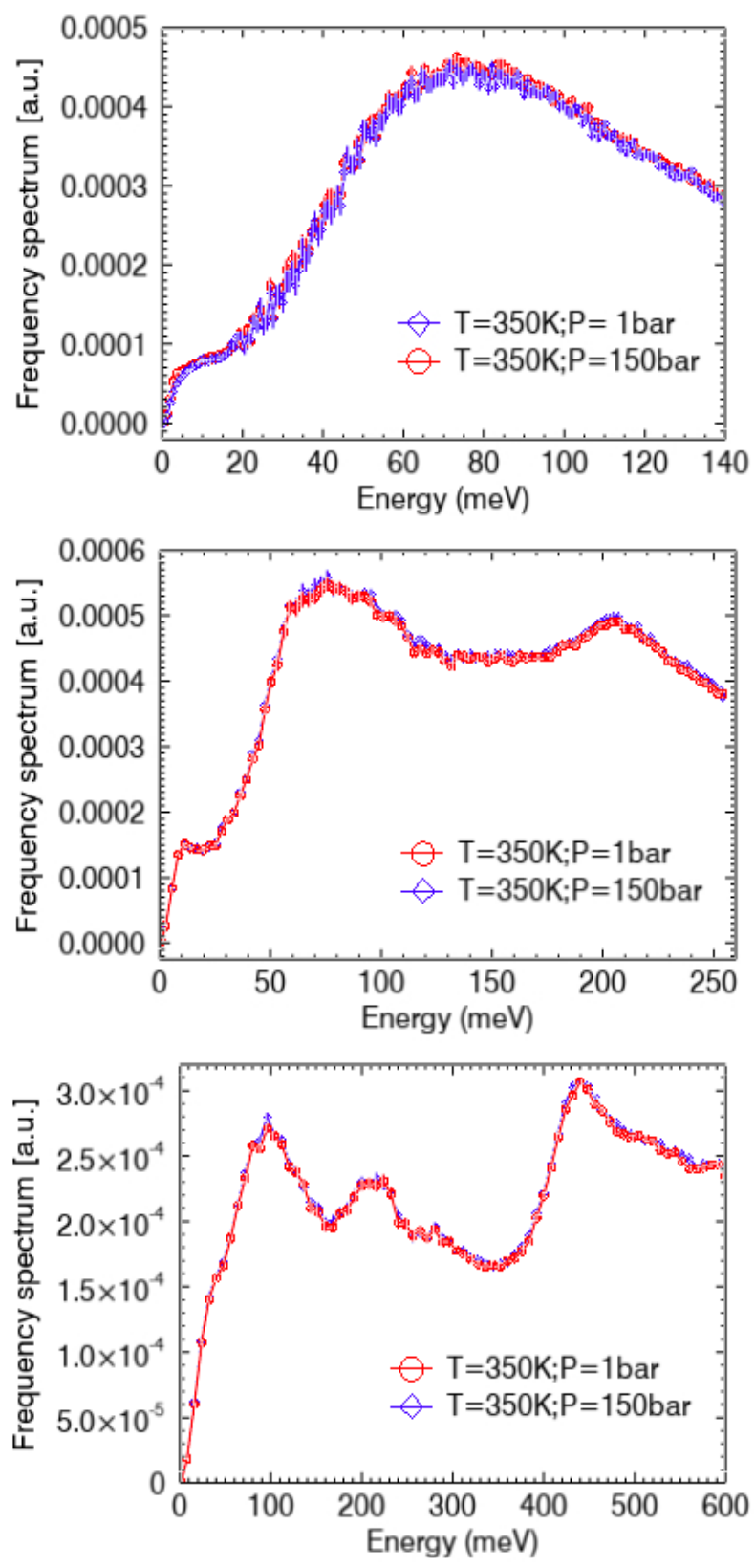

Figure 3. Variation of the frequency spectrum of light water as a function of pressure. The plots correspond to a temperature of $350 \mathrm{~K}$ and pressures $1 \mathrm{bar}$ and $150 \mathrm{bar}$. The upper, middle, and lower plots are for $E_{i} 160,280$ and $800 \mathrm{meV}$, respectively.

with $E_{i}$ as $160 \mathrm{meV}$ is to visualize the translation band around $9 \mathrm{meV}$, rotation band around $75 \mathrm{meV}$, the middle plot with $E_{i}$ as $280 \mathrm{meV}$ for the bending mode around 205 $\mathrm{meV}$, and the bottom plot with $E_{i}$ as $800 \mathrm{meV}$ to visualize the stretching mode. It was observed that pressure has a negligible impact on the frequency spectrum of light water. The translation band, rotation band, bending mode, and the stretching mode are unaffected by the change in pressure in the liquid state of water.

\subsection{Temperature dependence on the frequency spectrum}

The temperature dependence on the frequency spectrum of light water has been demonstrated by analyzing data at several temperatures between room temperature and 600 K. Figure 4 shows the variation of different energy modes in the frequency spectrum as a function of temperature. It
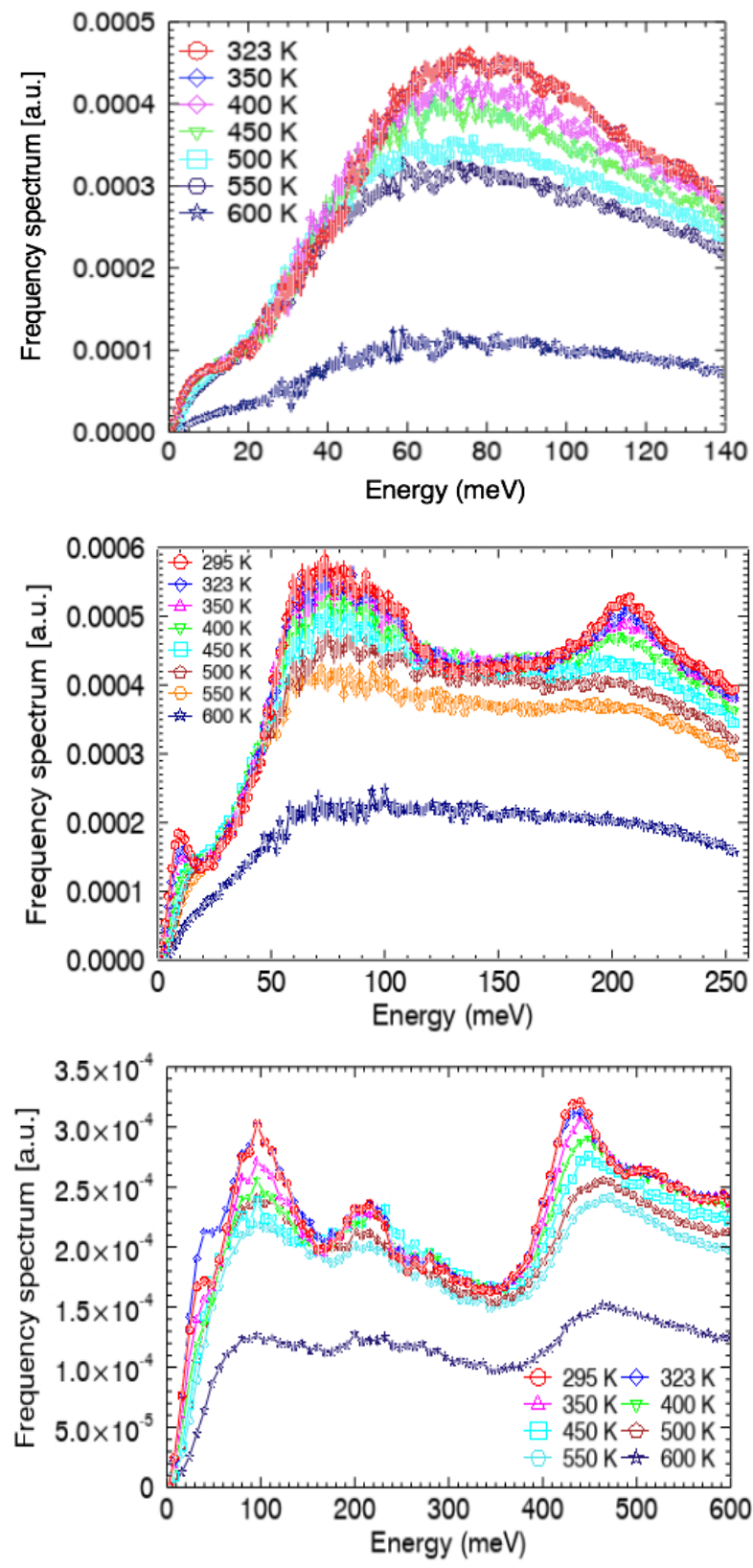

Figure 4. Variation of the frequency spectrum of light water as a function of temperature. The upper, middle and lower plots are for $E_{i} 160,280$ and $800 \mathrm{meV}$, respectively.

was observed that the position of the rotation band does not shift to lower energies with increasing temperature, and is peaked around $75 \mathrm{meV}$ at all the temperatures. 
However, the intensity of the rotation bands decreases with increasing temperature. This verifies the results observed at the ILL measurements for light water [5]. A similar observation was made for the bending mode around 205 $\mathrm{meV}$ for all the temperatures. However, for the stretching mode, it was observed that at room temperature the stretching mode is peaked around $436 \mathrm{meV}$ and shifts to around $470 \mathrm{meV}$ at $550 \mathrm{~K}$, as shown in Fig. 5.

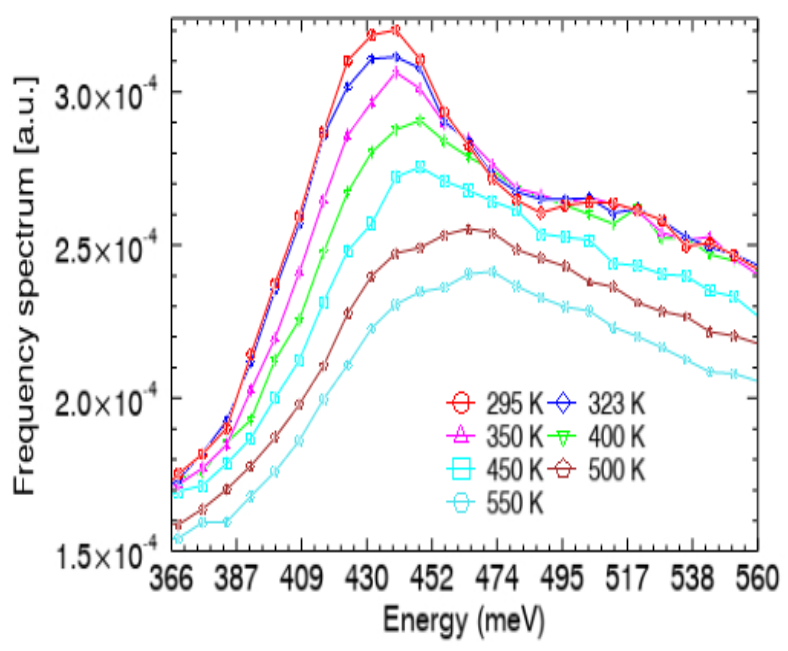

Figure 5. Frequency spectrum of light water obtained using $E_{i}=800 \mathrm{meV}$ to observe the temperature dependence of the stretching mode.

An interesting behavior of the frequency spectrum at $600 \mathrm{~K}$ (with a corresponding pressure of $150 \mathrm{bar}$ ) can be seen in Fig. 4 as this data lies close to the liquid-vapor saturation line. Any slight fluctuation in temperature and pressure would lead to a mixed state of water and water vapor, and hence justifies the strange behavior of the frequency spectrum. This observation implies a careful study of the TSL for accidental conditions where temperature and pressure change can cause huge changes in the light water cross sections.

\section{Conclusions}

High-resolution TOF inelastic measurements on light water at normal operating conditions of PWRs have been performed at the SEQUOIA spectrometer. Nine different sets of measurements at different incident neutron energies (8, $60,160,280$, and $800 \mathrm{meV}$ ) helped to investigate the full frequency spectrum domain. It was observed that pressure has a negligible impact on the frequency spectrum of light water in the liquid state. However, in a liquid-vapor mixed state (accidental scenario), its behavior is a matter of future research. The position of the translation band, rotation band and bending mode is unaffected by increasing temperature. However, the stretching mode tends to shift towards higher energies with increasing temperature. The obtained high-resolution frequency spectrum indicates improvement in the existing TSLs not only in the rotation band region, but also in the choice of the energy of the discrete oscillators as a function of temperature which as of now is assumed to be constant.

\section{Acknowledgements}

This research used resources at the Spallation Neutron Source, a DOE Office of Science User Facility operated by the Oak Ridge National Laboratory. The authors are grateful to Matthew Stone from SNS for his valuable contribution to the TOF data measurement and analysis.

\section{References}

[1] M. Mattes, J. Keinert, "Thermal Neutron Scattering Data for the Moderator Materials $\mathrm{H}_{2} \mathrm{O}, \mathrm{D}_{2} \mathrm{O}$ and $\mathrm{ZrH}_{x}$ in ENDF-6 Format and as ACE Library for MCNP(X) Codes" INDC(NDS)-0470, International Nuclear Data Committee (2005).

[2] B. Haywood, I. Thorson, "The Scattering Law for Light and Heavy Water at $20 \mathrm{C}$ and $150 \mathrm{C"}$, In: Proceedings of the Brookhaven Conference on Neutron thermalization, IAEA (1962).

[3] J. Marquez Damian, J. Granada and D. Malaspina, "CAB models for water: A new evaluation of the thermal neutron scattering laws for light and heavy water in ENDF- 6 format", Annals of Nuclear Energy, vol. 65, pp. 280-289 (2014).

[4] R. MacFarlane, D. Muir, "The NJOY Nuclear Data Processing System" LA-12470-M, Los Alamos National Laboratory, USA (1994).

[5] V. Jaiswal, "Theoretical and experimental approach towards generation of thermal scattering law for light water", Ph.D. thesis, University of Lille, Lille, (2018).

[6] I. Shvab and Richard J. Sadus, "Atomistic water models: Aqueous thermodynamic properties from ambient to supercritical conditions", Fluid Phase Equilibria, Vol. 407, 7-30 (2016).

[7] L. Leal, V. Jaiswal and A. Kolesnikov, "Highresolution neutron time-of-flight measurements for light water at the Spallation Neutron Source (SNS), Oak Ridge National Laboratory", ND2019 conference, Beijing, (2019).

[8] R.T. Azuah et al.,"DAVE: A comprehensive software suite for the reduction, visualization, and analysis of low energy neutron spectroscopic data", J. Res. Natl. Inst. Stan. Technol. 114, 341 (2009). 